https://doi.org/10.36396/MS.2019.15.4.001

\title{
Миокардиальная контрастная стресс-эхокардиография: современное состояние метода
}

\author{
М.А. САИАОВА, А.С. АТАБАЕВА, В.Н. ШИТОВ
}

Научно-исследовательский институт клинической кардиологии им. А.А. Мясникова ФГБУ «Национальный медицинский исследовательский центр кардиологии» МЗ РФ, 3-я Черепковская улица, А. 15а, 121552, Москва, Российская Федерация

\begin{abstract}
Резюме

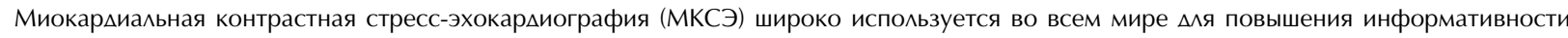
нагрузочной эхокардиографии в диагностике ишемии миокарда, а также мля улучшения качества визуализации у пациентов с плохим ультразвуковым окном. Использование современных ультразвуковых контрастных препаратов позволяет оценивать не только региональную и глобальную сократимость левого желудочка, но и проводить анализ перфузии миокарда. В настояшее время разработаны четкие рекомендации по проведению контрастных исследований в различных клинических группах пациентов. Высокий профиль безопасности ультразвуковых контрастных препаратов нового поколения Аоказан большим количеством клинических исследований, а сам метод завоевывает все больший интерес у специалистов.

Кмючевые слова: миокардиальная контрастная стресс-эхокардиография, ультразвуковые контрастные препараты, ишемия миокарда, пер фузия миокарда.
\end{abstract}

\section{Myocardial contrast stress-echocardiography: the current state of the method}

\author{
M.A. SAIDOVA, L.S. ATABAEVA, V.N. SHITOV
}

A.L. Myasnikov Insitute of Clinical Cardiology, Russian Cardiology Research and Production Complex, Ministry of Health of Russia, Moscow, Russia. 15a, 3d Cherepkovskaya street, 121552, Moscow, Russian Federation

\begin{abstract}
Summary
Myocardial contrast stress-echocardiography is widely used all over the world to improve image quality, reproducibility and reader confidence in the interpretation of the obtained images. The use of contrast in stress echocardiography has now extended beyond region wall motion to evaluation of myocardial perfusion. The clear recommendations for the use of ultrasound contrast have now been established in various patient populations. Safety of ultrasound contrast agents established in a large number of clinical trials.
\end{abstract}

Key words: myocardial contrast stress-echocardiography, ultrasound contrast agents, myocardial ischemia, myocardial perfusion.

Сведения об авторах:

Саихова Марина Абцулатиповна — профессор, м. м. н., руководитель отлела ультразвуковых методов исследования ФГБУ «НМИЦ кардиомогии» Минздрава России. E-mail: m.saidova@gmail.com

Атабаева Аина Салимовна - врач ультразвуковой диагностики, аспирант отАела ультразвуковых методов исследования ФГБУ «НМИЦ кардиологии» Минздрава России. E-mail: atabaeva_lina@mail.ru, +7 (925) 751-57-27 (автор, ответственный за переписку)

Шитов Виктор Николаевич - младший научный сотрудник отАела ультразвуковых методов исследования ФГБУ «НМИЦ кардиологии» Минзарава России. E-mail: vik.schitow@yandex.ru

\section{Введение}

Нагрузочная эхокардиография, или стресс-эхокардиография (стресс-ЭхоКГ), в настоящее время является одним из наиболее востребованных неинвазивных методов диагностики ишемии миокарда ввиду ее высокой информативности, безопасности и относительно низкой стоимости. В отношении оценки преходящей

(C) Комлектив авторов ишемии миокарда стресс-ЭхоКГ имеет существенные преимущества перед обычной нагрузочной электрокардиографической пробой в силу наличия комплекса эхокардиографических критериев, главным из которых является топическая диагностика зоны ишемии в бассейне пораженной коронарной артерии. Это нашло отражение в международных рекомендациях по диагностике хронической ишемической болезни сердца (ИБС), где стресс-ЭхоКГ прописана как один из основных неинвазивных методов диагностики ишемии миокарда [1]. 
Тем не менее у 20-30\% пациентов плохое качество визуализации не позволяет получить достоверную информацию о сократимости всех сегментов левого желудочка (ЛЖ), что приводит к необходимости проведения других диагностических методов, в том числе более дорогостоящих и менее безопасных для пациента [2]. Решением этой проблемы стало внедрение в клиническую практику новых ультразвуковых контрастных препаратов (УКП), которые при внутривенном введении с током крови заполняют не только правые, но и левые камеры сердца, проходя через микрососудистое русло легких [3].

K настоящему времени накоплен достаточно большой опыт проведения контрастных эхокардиографических исследований, который позволил Американскому эхокардиографическому обществу обновить в 2018 году рекомендации по клиническому применению ультразвуковых контрастных препаратов. В новых рекомендациях подтверждена их безопасность в различных группах пациентов, высокая информативность в определении прогноза больных и уменьшение стоимости обследования и лечения. Определены рекомендации в применении миокардиальной контрастной стресс-эхокардиографии (МКСЭ) для оценки перфузии миокарда, а также рассмотрены новые протоколы оптимизации изображения при проведении пробы, используемые различными производителями ультразвуковой аппаратуры [8].

\section{Характеристика ультразвуковых контрастных пре-} паратов

Современные ультразвуковые контрастные препараты представляют собой взвесь микропузырьков высокомолекулярного инертного газа (гексафторида серы или перфторпропана), покрытых эластичной оболочкой, состоящей из фосфолипидов или альбумина (рис. 1). Применение микропузырьков в качестве ультразвукового контрастного вещества базируется на способности пузырьков газа претерпевать объемную осцилляцию и стабильную кавитацию в ультразвуковом поле. Разница акустического импенданса между газом, выполняющим микропузырек, и окружающими его тканями обеспечивает усиление отраженного от таких пузырьков акустического сигнала. Использование инертных газов, обладающих более низкой растворимостью и диффузной способностью, чем воздух, позволяет увеличивать продолжительность жизни микропузырьков после внутривенной инъекции. Стабильность структуры достигается путем инкапсуляции микропузырьков в липидную или протеиновую оболочку, которая играет роль барьера, уменьшающего внешнюю диффузию газа и поверхностное натяжение микропузырьков [4]. Поскольку фаза увеличения объема больше фазы сжатия, продуцируемый микропузырьками сигнал (в отличие от сигнала от тканей) имеет нелинейный характер, что позволяет с помощью специальных алгоритмов выделить его, подавляя в то же время сигнал от тканей [5].

Для того чтобы безопасно преодолевать капиллярные сосуды легких, не вызывая эмболию, размер микропузырьков не должен превышать размера эритроцитов.
Рисунок 1. Строение укьтразвуковых контрастных препаратов: микропузырьки инертного газа, заключенные в биосовместимую оболочку.

Ацаптировано из статьи Jonathan R. Lindner, MD. [37]

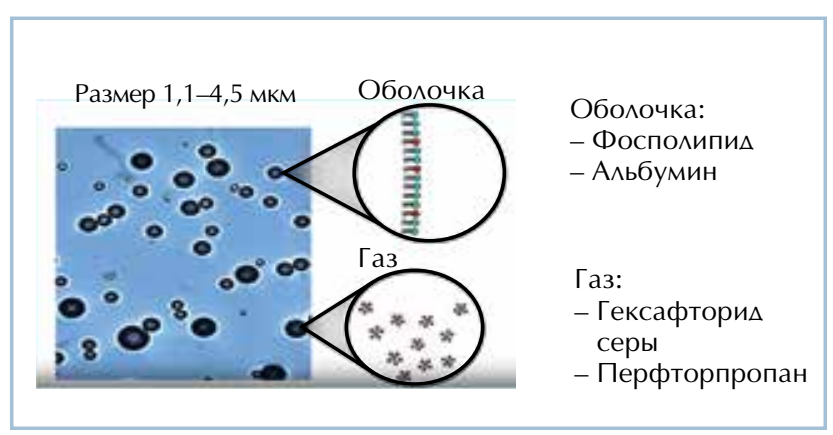

Микропузырьки, используемые в настоящее время, имеют диаметр от 1 до 6 мкм [6]. Безопасность современных ультразвуковых контрастных препаратов подтверждена большим количеством исследований, в том числе согласно последним рекомендациям [7, 8] сняты противопоказания у больных с легочной гипертензией, внутрисердечными шунтами и острым инфарктом миокарда. Микропузырьки газа не метаболизируются в почках или печени и выводятся из организма с дыханием в течение нескольких минут, что делает их самым безвредным контрастным веществом, известным на сегодняшний день. Тем не менее в некоторых случаях эти частицы распознаются системой комплимента как инородные и фагоцитируются, чем могут вызывать аллергические реакции. Нежелательные реакции в виде головных болей, кожных раздражений в месте инъекции, парестезий и других встречаются нечасто (1:1000), протекают легко, носят кратковременный характер и проходят спонтанно. Жизнеугрожающие случаи анафилактических реакций возникают крайне редко у 1 из 10 тыс. пациентов, в связи с чем в арсенале лаборатории должны быть все необходимые средства для купирования подобных состояний. На сегодняшний день противопоказаниями для использования УКП являются установленная аллергия на компоненты препарата, беременность и возраст менее 5 лет [4, 8].

\section{Способы введения ультразвуковых контрастных препаратов}

Существует два возможных варианта введения УКП: внутривенная инфузия или небольшие болюсы с последующим медленным введением физиологического раствора. Оба метода имеют как свои преимущества, так и недостатки. При болюсном введении контрастирование наступает быстро и интенсивно, но также быстро и спадает, так как концентрация микропузырьков в пуле крови нестабильна во времени. При инфузионном введении контрастирование полости и стенок миокарда более устойчивое, однако этот способ требует использования специальных инфузоматов. Таким образом, метод болюсного введения УКП наиболее легок в применении, не требует дополнительного оборудования 
Рисунок 2. Апикальная хвухкамерная позиция: изображения в конце диастолы (слева) и в конце систолы (справа). Показана наикучшая визуализашия зоны нарушения мокальной сократимости по нижней стенке $\Lambda Ж$ (указано стрелками) при использовании изображений с очень низким механическим инцексом

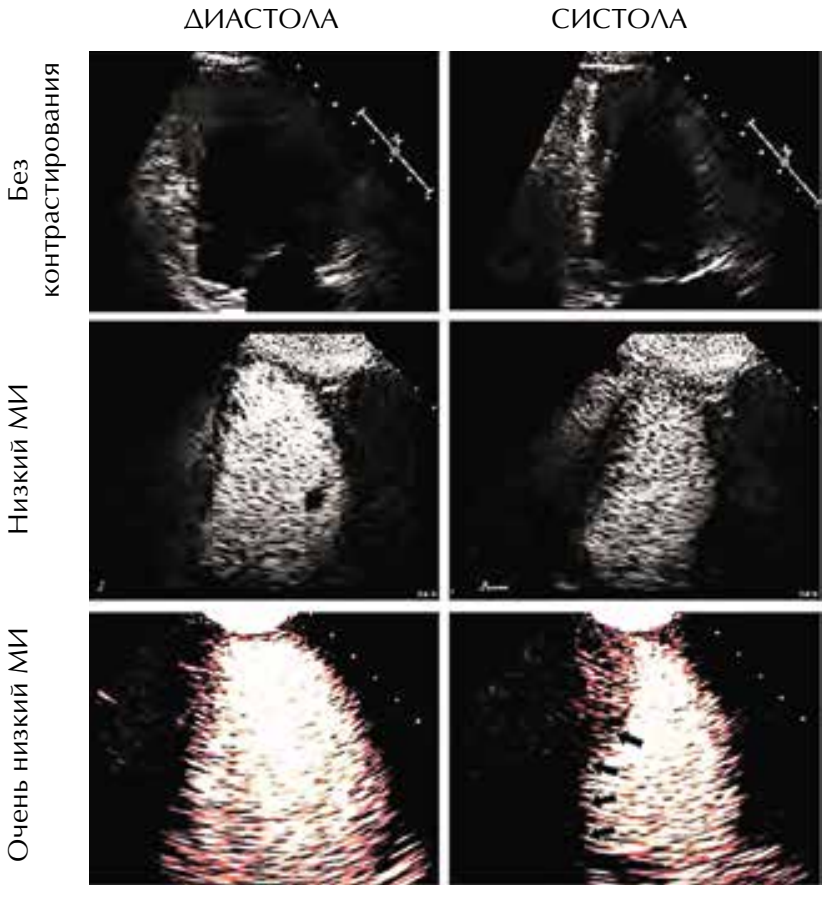

и подходит для решения большинства диагностических задач [7].

Оптимизация изображения при проведении МКСЭ

Проведение сканирования в ходе исследования также имеет ряд особенностей. Степень колебания микропузырьков частично зависит от интенсивности ультразвукового поля. Показателем интенсивности передаваемого ультразвукового сигнала является механический индекс (МИ), который составляет >0,8 для большинства неконтрастных исследований [7]. При длительном и мощном акустическом воздействии происходит деструкция микропузырьков, поэтому для сканирования при контрастной ЭхоКГ используют режимы с низким МИ [5]. Во всех современных приборах имеются специальные настройки сканера в виде пресетов. Один из них - это режим с низким МИ $(<0,3)$ и использованием второй тканевой гармоники. Этот режим позволяет ослабить сигнал от тканей, уменьшить разрушение микропузырьков и добиться контрастирования полости ЛЖ для визуализации границы эндокарда и оценки региональной сократимости. Однако данный режим имеет некоторые особенности. В связи с относительно высокой мощностью сигнала происходит довольно быстрое разрушение микропузырьков, что ухудшает контрастирование апикальных сегментов и требует дополнительного введения препарата. В то же время слишком большая концентрация препарата в верхушке ослабляет сигнал от базальных сегментов. Другой режим предполагает использование излучения с очень низким МИ $(<0,2)$ в сочетании с инверсией фазы или модуляцией амплитуды импульса. Это позволяет отсечь сигнал от тканей и усилить сигнал от микропузырьков, заполняющих полость ЛЖ и микроциркуляторное русло [8]. К преимуществам этого режима относятся меньшая скорость разрушения препарата, снижение количества артефактов, улучшение визуализации границы эндокарда во всех сегментах, а также контрастирование самого миокарда (рис. 2). Поэтому именно данный режим рекомендован для оценки не только сократимости, но и перфузии миокарда как в покое, так и при стресс-ЭхоКГ [8].

\section{Методика проведения МКСЭ}

Протокол МКСЭ принципиально не отличается от обычного, используемого при стандартной стресс-ЭхоКГ (рис. 3). Введение препарата с последующей записью изображений для оценки сократимости осуществляется исходно и на максимуме нагрузки,

Рисунок 3. Протокол проведения миокардиальной контрастной стресс-ЭхоКГ

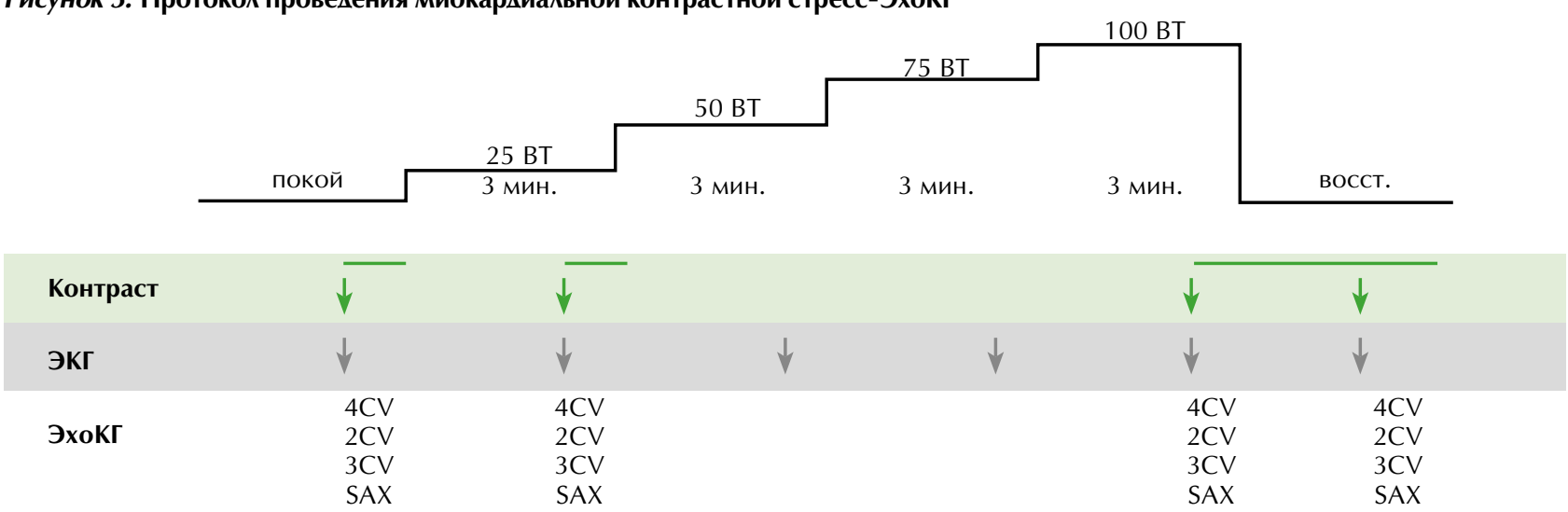

4CV - четырехкамерная апикальная позиция, 2CV - двухкамерная апикальная позиция, 3CV - трехкамерная апикальная позиция, SAX - парастернальная позиция по короткой оси ЛЖ. 
иногда также на одной из промежуточных ступеней и в периоде восстановления. Если в протокол включена оценка перфузии, то это делается исходно и в течение 1,5 минуты после прекращения нагрузки, предпочтительна запись стабильных изображений на задержке дыхания [7].

Ключевым моментом для получения реальной диагностической информации при МКСЭ является уровень подготовки специалиста, который включает в себя обучение методике в крупных центрах с большим опытом подобных исследований. Необходимо выполнить 100 исследований под контролем эксперта и в дальнейшем проводить не менее 50 подобных исследований в год [7].

\section{Оценка региональной сократимости миокарда}

Как уже отмечалось, применение МКСЭ наиболее актуально у пациентов с так называемым плохим ультразвуковым окном. Контрастирование полости ЛЖ при исследовании с низким уровнем акустической мощности позволяет достичь более четкой визуализации границ эндокарда, что повышает чувствительность, специфичность и диагностическую достоверность метода как при проведении стрессЭхоКГ с физической нагрузкой, так и с фармакологическими пробами [9].

В 2008 году были опубликованы результаты исследования OPTIMIZE, которое показало, что применение УКП увеличивало диагностическую достоверность стресс-ЭхоКГ у пациентов с неоптимальной визуализа-

Рисунок 4. Четырехкамерная апикамьная позиция: сравнение стандартной стресс-ЭхоКГ и миокардиахьной контрастной стресс-ЭхоКГ (МКСЭ) у пациента с неуцовлетворительной визуализачией эндокарда $\Lambda Ж$ При введении контрастного препарата определяется гипокинез по зацнебоковой стенке $\Lambda Ж$ (указан стрелками), который не определяется при станцартной стресс-ЭхоКГ

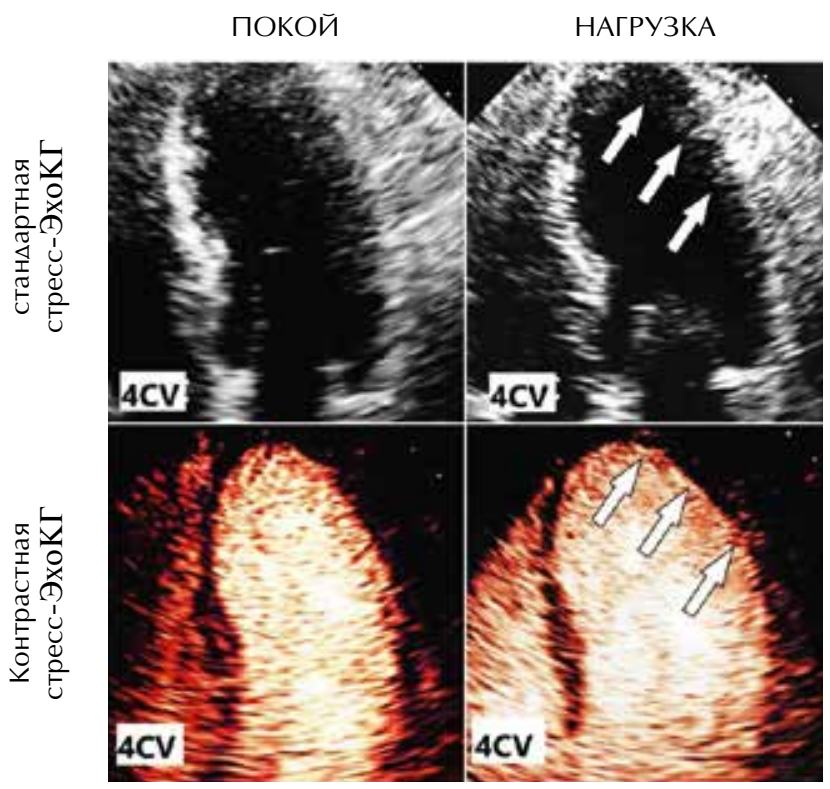

цией с 36 до 74\%. Однако не было выявлено достоверного различия у пациентов с хорошей визуализацией всех сегментов ЛЖ [10]. По данным другого исследования, из 839 пациентов с различным качеством визуализации использование контрастного препарата при стресс-ЭхоКГ улучшило детекцию границ эндокарда как в покое, так и на пике нагрузки в 99,3\% случаев, снижая тем самым вариабельность интерпретации полученных данных [11].

По различным данным литературы, чувствительность и специфичность стандартной стресс-ЭхоКГ в среднем составляют около 82 и $78 \%$ соответственно, в то время как при использовании контрастного вещества эти показатели увеличиваются до 93 и 80\% [12]. На рисунке 4 приведена сравнительная характеристика стандартной стресс-ЭхоКГ и МКСЭ в покое и на пике нагрузки.

Использование МКСЭ у пациентов с плохим качеством визуалиации отражается и на экономической стороне вопроса, так как стоимость самого контрастного препарата более чем компенсируется экономией, полученной за счет сокращения повторных исследований и более низкой частоты ложноположительных и ложноотрицательных результатов [13]. В частности, по сравнению с нагрузочной ЭКГ и однофотонной эмиссионной компьютерной томографией (ОЭКТ) МКСЭ реже требует проведения дополнительных уточняющих методов исследования, что коррелирует со значительно меньшими экономическими затратами [14].

Согласно Европейской ассоциации по сердечно-сосудистой визуализации, применение контрастного препарата при стресс-ЭхоКГ показано всем больным с неудовлетворительной визуализацией двух или более смежных сегментов ЛЖ (уровень доказательности I А) [7].

\section{Качественная оценка перфузии миокарда}

Еще одним фактором, приводящим к повышению чувствительности МКСЭ, является раннее обнаружение субэндокардиальной ишемии, проявляющейся снижением перфузии миокарда, в том числе при сохранной сократимости в этой области $[15,16]$. Данный феномен обусловлен каскадом реакций, происходящим в ишемизированном участке миокарда, благодаря которому снижение перфузии предшествует появлению нарушения сократительной функции [17]. Оценка перфузии проводится на основании повторного накопления УКП в миокарде после разрушения микропузырьков высокоэнергетическим импульсом (Flash с МИ = 0,8-1,2). В норме повторное заполнение миокарда контрастным веществом после его деструкции должно происходить менее чем за 5 секунд в покое и менее чем за 2 секунды во время нагрузки. Замедленное заполнение свидетельствует о нарушении перфузии, причем это не всегда сопровождается нарушением сократительной функции [18].

В настоящее время остается открытым вопрос о целесообразности реваскуляризации пациентов со снижением перфузии миокарда во время нагрузки 
Рисунок 5. Трехкамерная апикальная позиция: протокол оценки перфузии миокарца при МКСЭ. В покое повторное заполнение миокарда контрастным препаратом после импульса с высоким механическим инцексом (flash) полностью происходит за 4 секунды. Во время нагрузки наблюдается замедленное повторное накопление УКП области зацнебоковой стенки АЖ (указано стрелками), что говорит о наличии субэндокардиальной ишемии в этом участке

перех flash
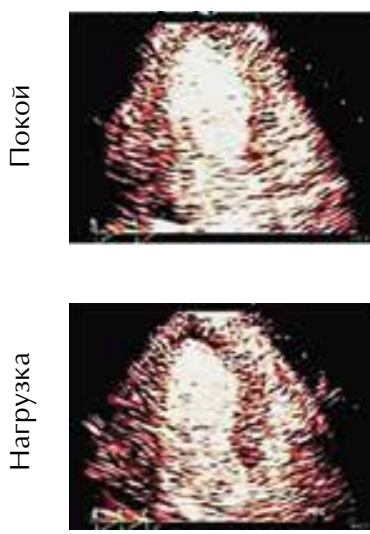

сразу после flash
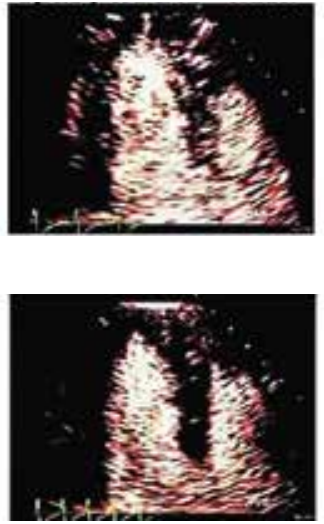

через 1 секунду
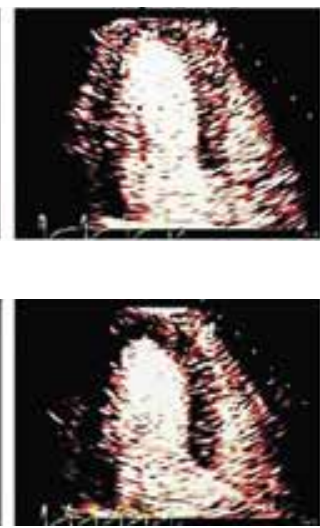

через 2 секунды
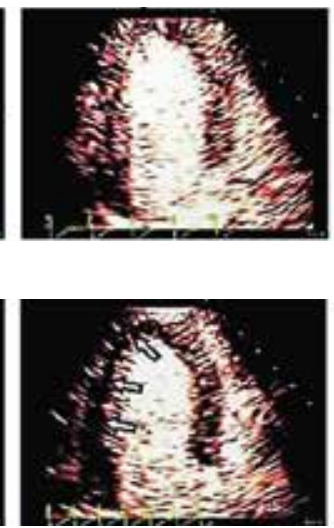

через 14 секун $\Delta$
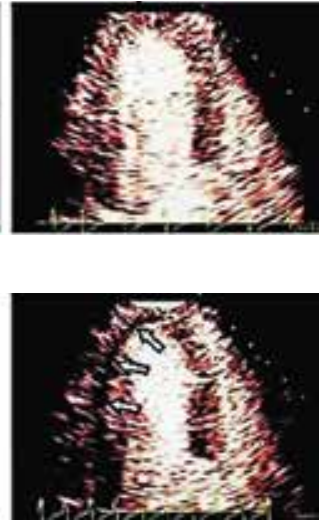

при отсутствии нарушения локальной сократимости. По данным нерандомизированного ретроспективного исследования, выполненного в 2017 году, проведение чрескожного коронарного вмешательства у таких пациентов было ассоциировано с более высоким риском смерти и тяжелых кардиальных событий по результатам трехлетнего и шестилетнего наблюдений, чем у пациентов, находившихся на медикаментозной терапии. Однако необходимы дальнейшие проспективные исследования в этой области [19].

В последние годы проведены работы, в которых продемонстрировано более высокое прогностическое значение оценки перфузии миокарда в сочетании с сократимостью по сравнению с контрастной стресс-ЭхоКГ, где оценивалась только сократимость миокарда при разных видах нагрузки: с велоэргометрией [20], тредмил-тестом [21], пробой с добутамином [22] и дипиридамолом [23, 24] у больных с ИБС. В каждом из этих исследований замедленное повторное накопление контрастного препарата при нормальной сократимости наблюдалось у значительного процента пациентов и являлось независимым предиктором смерти и развития инфаркта миокарда.

В кардиологии для изучения перфузии миокарда в покое и при нагрузке наиболее широко применяется однофотонная эмиссионная компьютерная томография (ОЭКТ). Несколько работ показали, что МКСЭ имеет не только более высокую специфичность, но и чувствительность по сравнению с ОЭКТ в выявлении гемодинамически значимых стенозов коронарных артерий у пациентов с различными анатомическими вариантами поражения [25], а также имеет более высокую прогностическую ценность [26]. Кроме того, МКСЭ имеет существенные преимущества в оценке перфузии миокарда при полной блокаде левой ножки пучка Гиса и артифициальном ритме, так как при ОЭКТ в этой группе пациентов в $80 \%$ случаев выяв- ляются дефекты перфузии передне-перегородочной, верхушечной локализаций в отсутствие поражения коронарных артерий [27]. Помимо лучшей разрешающей способности и возможности оценивать глобальную, региональную сократимость и диастолическую функцию ЛЖ к преимуществам МКСЭ также относятся меньшая стоимость исследования и отсутствие лучевой нагрузки [8].

Данные МКСЭ сопоставлялись со значениями фракционного резерва кровотока (ФРК) у пациентов с пограничными стенозами коронарных артерий (50-80\%). Так, в 93\% случаев при наличии значимого снижения ФРК были выявлены признаки субэндокардиальной ишемии в бассейне соответствующих коронарных артерий, по данным МКСЭ. При этом у 57\% пациентов со значениями ФРК более 0,8 наблюдалось снижение перфузии миокарда в соответствующих бассейнах кровоснабжения. В этой группе дальнейшее наблюдение было возможно у 26 из 27 пациентов. Из них 16 пациентам (59\%) была проведена реваскуляризация миокарда в связи с сохранением клиники стенокардии II-III функционального класса (ФК), несмотря на медикаментозную терапию, в то время как 10 пациентов остались бессимптомными на фоне консервативной терапии. Отсутствие достоверной корреляции между двумя методами может объясняться тем, что при оценке ФРК измеряется градиент давления между престенотическим и постстенотическим участками артерии, но не учитывается влияние капиллярной резистентности, которая является основным регулятором коронарного кровотока в момент стресса. Другими словами, снижения перфузии, обнаруживаемые при МКСЭ, могут существовать до появления разности давлений в месте стеноза. Однако необходимы дальнейшие исследования в этом направлении [28].

Анализ перфузии миокарда также может применяться для оценки реперфузионной терапии при инфаркте 
миокарда [29] или для оценки жизнеспособоности миокарда [30].

МКСЭ с оценкой перфузии должна рассматриваться у всех пациентов, которым проводится фармакологическая проба и у пациентов высокого риска ИБС при проведении пробы с физической нагрузкой (уровень доказательности I А) [7].

\section{Количественная оценка перфузии миокарда, по данным МКСЭ}

При использовании специальных программ возможен количественный анализ скорости заполнения микроциркуляторного русла миокарда контрастным препаратом в покое и на нагрузке с расчетом резерва миокардиального кровотока. В одной из недавно опубликованных работ продемонстрировано дополнительное прогностическое значение этого показателя в сравнении с обычной, качественной, оценкой перфузии и сократимости [18].

Для количественной оценки перфузии миокарда были разработаны специальные подходы, основанные на измерении двух показателей перфузии миокарда: количества единиц, активно перфузирующихся через микроциркуляторное русло в любой момент времени (микрососудистый объем крови - МОК) и скорости потока крови через эти микрососудистые единицы [31]. Измерение этих параметров основано на способности ультразвукового поля влиять на целостность микропузырьков. Импульсы с высоким МИ $(>0,8)$ разрушают микропузырьки в микрососудистом русле. При этом анализ времени повторного накопления контрастного препарата в микрососудистом русле может быть использован для оценки скорости и степени накопления сигнала микропузырьков, отражающих скорость микрососудистого потока и МОК соответственно. Эту процедуру наиболее предпочтительно проводить с использованием непрерывной инфузии УКП, чтобы достичь стабильной концентрации микропузырьков в пуле крови. Проводить анализ рекомендуется в конце систолы (для устранения сигнала от миокардиальных сосудов) [32, 33]. Небольшие болюсы УКП с последующим введением физиологического раствора также могут создавать период времени после каждой инъекции, когда концентрация контрастного препарата равномерна, позволяя проводить количественный анализ перфузии [34, 35].

Количественная оценка перфузии может быть выражена уравнением у $=$ A $\left(1-\mathrm{e}^{-\beta \mathrm{t}}\right)$, где у - интенсивность сигнала в любой момент времени t после высокоэнергетического импульса Flash, A - интенсивность

Рисунок 6. Количественная оценка перфузии миокарда. Первое изображение после серии импульсов с высоким МИ (flash) имлюстрирует отсутствие сигнала от миокарда ввицу полной деструкции микропузырьков. Последуюшие кацры цемонстрируют постепенное увеличение интенсивности сигнала при заполнении микроширкуяторного русла миокарда микропузырьками. Ниже показан график скорости потока эритроцитов (FluxRBC) из константы скорости и микрососудистого объема кровотока (интенсивность сигнала плато A). Ацаптировано из статьи T.R. Porter et al. [8]

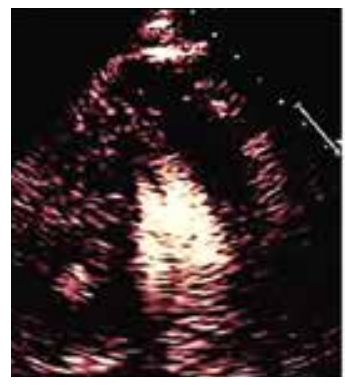

постьеструкция

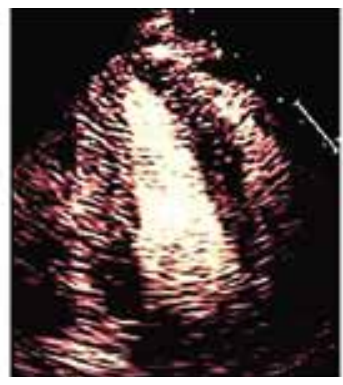

$t_{1}$

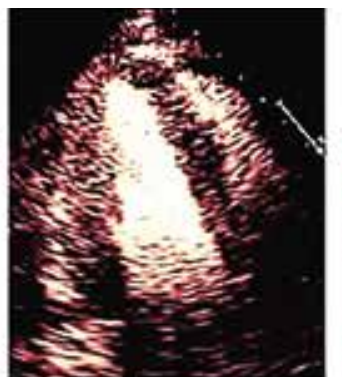

$\mathrm{t}_{2}$

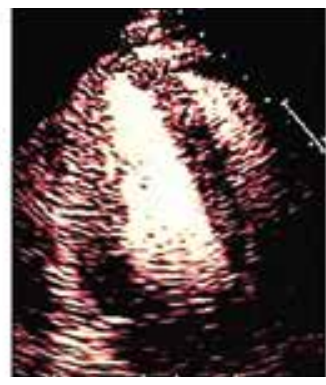

$t_{3}$

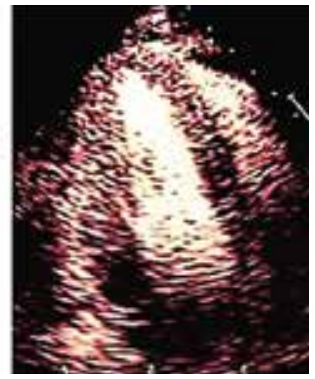

$t_{5}$

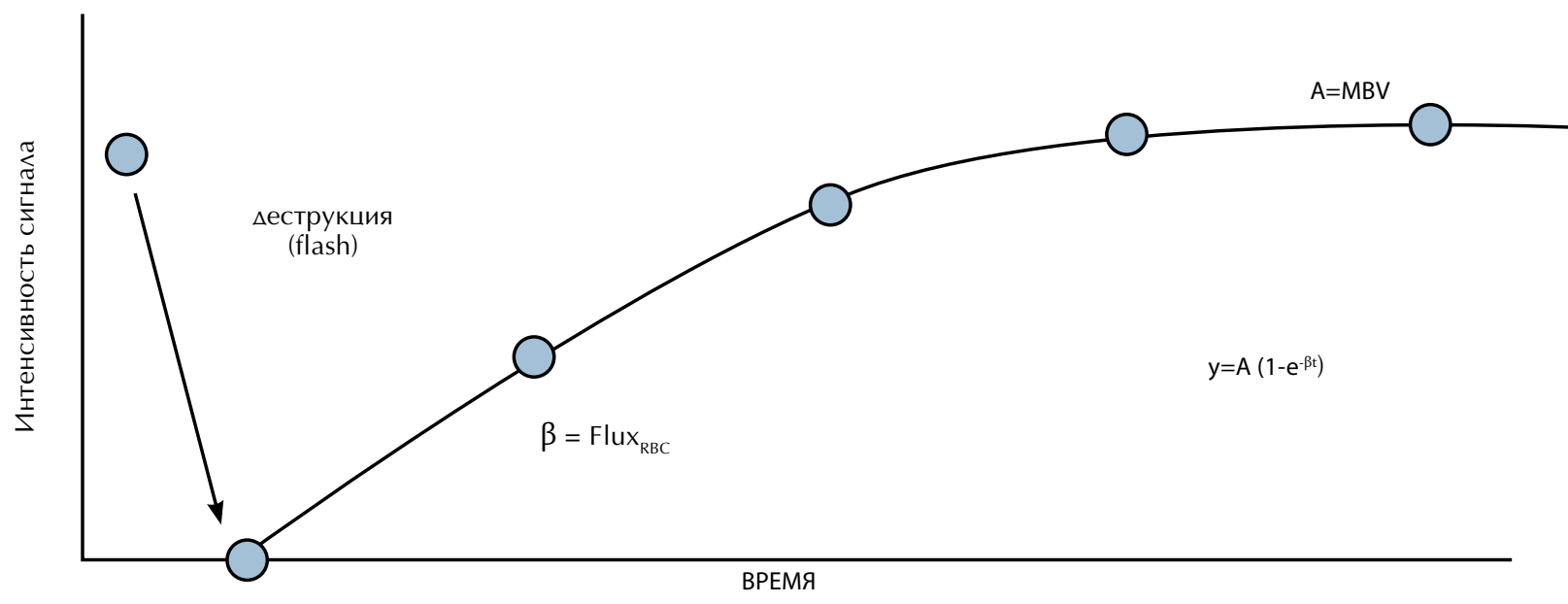


сигнала плато, отражающая относительный МOK, а $\beta\left(\mathrm{sec}^{-1}\right)$ - константа, отражающая скорость потока микропузырьков через микроциркуляторное русло [31].

Отношение интенсивности плато (нагрузка/покой) не показало эффективности в диагностике ИБС, однако коэффициент $\beta$ или значение $\mathrm{A} x \boldsymbol{\beta} \geq 2$ имеют прогностическое значение в дифференциации нормального и аномального резерва миокардиального кровотока [36].

Важным ограничением такой количественной оценки является тот факт, что небольшая зона тяжелой ишемии в сегменте может приводить к идентичным данным, что и более распространенная зона умеренной ишемии. Соответственно, рекомендуется количественный анализ сопровождать качественной оценкой пространственных перфузионных нарушений как по числу вовлеченных сегментов, так и по характеру поражения: трансмурального или субэндокардиального [8].

В настоящее время количественный подход к изучению перфузии миокарда не рекомендован для широкого клинического применения ввиду отсутствия широкодоступного и удобного для использования программного обеспечения.

\section{ЛИТЕРАТУРA/REFERENCES}

1. G. Montalescot, U. Sechtem, S. Achenbach et al. 2013 ESC guidelines on the management of stable coronary artery disease: The Task Force on the management of stable coronary artery disease of the European Society of Cardiology. European Heart Journal. Volume 34, Issue 38, 7 October 2013, Pages 2949-3003.

2. Grayburn P.A., Mulvagh Crouse L. Left ventricular opacification at rest and during stress. Am J Cardiol. 2002; 90(suppl):21J-27J.

3. Thomas R. Porter, MD et al. Guidelines for the Cardiac Sonographer in the Performance of Contrast Echocardiography: A Focused Update from the American Society of Echocardiography. August 2014, Volume 27, Issue 8, Pages 797-810.

4. Jonathan R. Lindner, M.D. et al. A Practical Approach to Contrast Echocardiography. American college of cardiology, Jul 10, 2017; vol. 3 no. 2 212-218.

5. Lindner, Jonathan R. et al. Phase-conversion nanoparticle contrast agents: Do good things come in small packages? Circulation: Cardiovascular Imaging. Jan 1, 2016. Volume 9, Issue number 1, e004374.

6. Lindner, Jonathan R. et al. Microvascular rheology of Definity microbubbles after intra-arterial and intravenous administration. Journal of the American Society of Echocardiography. Volume 15, Issue 5, 396 - 403.

7. Roxy Senior, Harald Becher, Mark Monaghan, Luciano Agati et al. Clinical practice of contrast echocardiography: recommendation by the European Association of Cardiovascular Imaging (EACVI) 2017. European Heart Journal - Cardiovascular Imaging (2017). Volume 18, Issue 11, 1 November 2017, Pages 1205-1205af.

8. Thomas R. Porter, MD, Sharon L. Mulvagh, MD et al. Clinical Applications of Ultrasonic Enhancing Agents in Echocardiography: 2018 American Society of Echocardiography Guidelines Update. Journal of the American Society of Echocardiography. March 2018. Volume 31 Number 3; 241-274.

9. Mulvagh S.L., Rakowski H., Vannan M.A., Abdelmoneim S.S., Becher H., Bierig S.M. et al. ASE consensus statement on the clinical applications of ultrasonic contrast agents in echocardiography. JAm Soc Echocardiogr. 2008;21:1179-201.

10. Plana J.C., Mikati I.A., Dokainish H., Lakkis N., Abukhalil J., Davis R. et al. A randomized cross-over study for evaluation of the effect of image optimization with contrast on the diagnostic accuracy of dobutamine echocardiography in coronary artery disease: the OPTIMIZE trial. JACC Cardiovasc Imaging. March 2008; Volume 1, Issue 2, 145-152.

11. Shah B.N., Balaji G., Alhajiri A., Ramzy I.S., Ahmadvazir S., Senior R Incremental diagnostic and prognostic value of contemporary stress echocardiography in a chest pain unit: mortality and morbidity outcomes from a real-world setting. Circ Cardiovasc Imaging. 2013;6:202-9

12. Stress Echocardiography with Contrast for the Diagnosis of Coronary Artery Disease. An Evidence-Based Analysis. Ont Health Technol Assess Ser. 2010; 10(10): 1-59.

\section{Зак^ючение}

Использование ультразвукового контрастного препарата при стресс-ЭхоКГ значительно улучшает визуализацию границ эндокарда ЛЖ у пациентов с плохим ультразвуковым окном. Помимо этого, МКСЭ позволяет выявлять дефекты перфузии миокарда как в покое, так и во время нагрузки, повышая тем самым чувствительность стресс-ЭхоКГ в выявлении обратимой ишемии миокарда вне зависимости от качества визуализации. К другим важным преимуществам МКСЭ по сравнению с другими конкурирующими методами исследования можно отнести относительно небольшую стоимость, высокое пространственное разрешение, возможность оценки ишемии миокарда в режиме реального времени, а также отсутствие ионизирующего излучения. По этим причинам МКСЭ как наиболее безопасный и высокоинформативный неинвазивный визуализирующий метод, вероятно, станет предпочтительным для более точной оценки ишемии миокарда в будущем.

\section{Авторы заявляют об отсутствии конфликта интересов.}

Поступила 30.07.2019

Принята в печать 27.09.2019

13. Thanigaraj S., Nease R.F., Schechtman K.B., Wade R.L., Loslo S., Perez J.E.: Use of contrast for image enhancement during stress echocardiography is cost-effective and reduces additional diagnostic testing. Am J Cardiol. 2001, 87: 1430-1432. 10.1016/S0002-9149(01)01573-9.

14. Zacharias K., Ahmed A., Shah B.N., Gurunathan S., Young G., Acosta D. et al. Relative clinical and economic impact of exercise echocardiography vs. exercise electrocardiography, as first line investigation in patients without known coronary artery disease and new stable angina: a randomized prospective study. Eur Heart J Cardiovasc Imaging. 2017;18: 195-202.

15. Xie F., Dodla S., O'Leary E., Porter T.R. Detection of subendocardial ischemia in the left anterior descending coronary artery territory with realtime myocardial contrast echocardiography during dobutamine stress echocardiography. JACC Cardiovasc Img. 2008;1:271-8.

16. Porter T.R., Smith L.M., Wu J., Thomas D., Haas J.T., Mathers D.H. et al Patient outcome following 2 different stress imaging approaches. $\mathrm{J} \mathrm{Am} \mathrm{Coll}$ Cardiol. 2013;61:2246-455.

17. Leong-Poi H., Rim S.J., Le D.E., Fisher N.G., Wei K., Kaul S. Perfusion versus function: the ischemic cascade in demand ischemia: implications of single-vessel versus multivessel stenosis. Circulation. 2002;105:987-92.

18. Angele A. A. Mattoso, Jeane M. Tsutsui et al. Prognostic value of dobutamine stress myocardial perfusion echocardiography in patients with known or suspected coronary artery disease and normal left ventricular function PLoS ONE. 2017;12(2):e0172280.

19. Gaibazzi N., Porter T., Lorenzoni V. et al. Effect of Coronary Revascularization on the Prognostic Value of Stress Myocardial Contrast Wall Motion and Perfusion Imaging. J Am Heart Assoc. 2017 May 31;6(6). pii: e006202. doi: 10.1161/JAHA.117.006202.

20. Miszalski-Jamka T., Kuntz-Hehner S., Schmidt H., Peter D., MiszalskiJamka K., Hammerstingl C. et al. Myocardial contrast echocardiography enhances long-term prognostic value of supine bicycle stress two dimensional echocardiography. JAmSoc Echocardiogr 2009;22:1220-7.

21. Porter T.R., Smith L.M., Wu J., Thomas D., Haas J.T., Mathers D.H. et al Patient outcome following 2 different stress imaging approaches. J Am Coll Cardiol. 2013;61:2246-455.

22. Dolan M.S., Gala S.S., Dodla S., Abdelmoneim S.S., Xie F., Cloutier D. et al. Safety and efficacy of commercially available ultrasound contrast agents for rest and stress echocardiography: a multicenter experience. J Am Coll Cardiol. 2009;53:32-8.

23. Gaibazzi N., Reverberi C., Lorenzoni V., Molinaro S., Porter T.R. Prognostic value of high-dose dipyridamole stress myocardial contrast perfusion echocardiography. Circulation. 2012;126:1217-24.

24. Thomas D., Xie F., Smith L.M., O'Leary E., Smith K., Olson J. et al Prospective randomized comparison of conventional stress echocardiography 
and real time perfusion stress echocardiography in detecting significant coronary artery disease. J Am Soc Echocardiogr. 2012;25:1207-14.

25. Senior R., Moreo A., Gaibazzi N., Agati L. et al. Comparison of sulfur hexafluoride microbubble (SonoVue)-enhanced myocardial contrast echocardiography with gated single-photon emission computed tomography for detection of significant coronary artery disease: a large European multicenter study. J Am Coll Cardiol. 13 Jun 2013, 62(15):1353-1361.

26. DawsonD., KaulS., PetersD., RinkevichD., SchnellG., BelcikJ.T.etal. Prognostic value of dipyridamole stress myocardial contrast echocardiography: comparison with single photon emission computed tomography. J Am Soc Echocardiogr. 2009; 22:954 \pm 960 . doi: 10.1016/j.echo.2009.04.034 PMID: 19553084 .

27. Hayat S.A., Dwivedi G., Jacobsen A., Lim T.K., Kinsey C., Senior R. Effects of left bundle branch block on cardiac structure, function, perfusion, and perfusion reserve: implications for myocardial contrast echocardiography versus radionuclide perfusion imaging for the detection of coronary artery disease. Circulation. 2008;117:1832-41.

28. Wu J., Barton D., Xie F., O’Leary E., Steuter J., Pavlides G. et al. Comparison of fractional flow reserve assessment with demand stress myocardial contrast echocardiography in angiographically intermediate coronary stenosis. Cir Cardiovasc Imaging. 2016;9:e004129.

29. Janardhanan R., Moon J.C., Pennell D.J., Senior R. Myocardial contrast echocardiography accurately reflects transmurality of myocardial necrosis and predicts contractile reserve after acute myocardial infarction. Am Heart J. 2005; 149:355-62.

30. Balcells E., Powers E.R., Lepper W., Belcik T., Wei K., Ragosta M. et al. Detection of myocardial viability by contrast echocardiography in acute infarction predicts recovery of resting function and contractile reserve. $J \mathrm{Am}$ Coll Cardiol. 2003;41:827-33.
31. Wei K., Jayaweera A.R., Firoozan S., Linka A., Skyba D.M., Kaul S. Quantification of myocardial blood flow with ultrasound-induced destruction of microbubbles administered as a constant venous infusion. Circulation. 1998;97:473-83.

32. Leong-Poi H., Le E., Rim S.J., Sakuma T., Kaul S., Wei K. Quantification of myocardial perfusion and determination of coronary stenosis severity during hyperemia using real-time myocardial contrast echocardiography. J Am Soc Echocardiogr. 2001;14:1173-82.

33. Leong-Poi H., Swales J., Jayaweera A.R., Bin J.P., Kaul S., Lindner J.R. Effect of microbubble exposure to ultrasound on quantitation of myocardial perfusion. Echocardiography. 2005;22:503-9.

34. Gaibazzi N., Rigo F., Reverberi C. Detection of coronary artery disease by combined assessment of wall motion, myocardial perfusion and coronary flow reserve: a multiparametric contrast stress-echocardiography study. $\mathrm{J} \mathrm{Am} \mathrm{Soc}$ Echocardiogr. 2010;23:1242-50.

35. Gaibazzi N., Reverberi C., Lorenzoni V., Molinaro S., Porter T.R. Prognostic value of high-dose dipyridamole stress myocardial contrast perfusion echocardiography. Circulation. 2012;126:1217-24.

36. Abdelmoneim S.S., Dhoble A., Bernier M., Erwin P.J., Korosoglou G., Senior R. et al. Quantitative myocardial contrast echocardiography during pharmacological stress for diagnosis of coronary artery disease: a systematic review and meta-analysis of diagnostic accuracy studies. Eur $J$ Echocardiogr. 2009; 10:813-25.

37. Jonathan R. Lindner, MD. Contrast Echocardiography: The Bubbles and How We Image Them. Knight Cardiovascular Institute Oregon Health \& Science University Portland. Oregon, 2016. 